\title{
Experimental removal of introduced slider turtles offers new insight into competition with a native, threatened turtle
}

\author{
Max R Lambert ${ }^{\text {Corresp., } 1}$, Jennifer M McKenzie ${ }^{2}$, Robyn M Screen ${ }^{3}$, Adam G Clause ${ }^{4}$, Ben J Johnson ${ }^{5}$, Genevieve G \\ Mount $^{6}$, H Bradley Shaffer ${ }^{7}$, Gregory B Pauly ${ }^{4}$ \\ 1 Department of Environmental Science, Policy, and Management, UC Berkeley, Berkeley, California, United States of America \\ 3 Department of Biology, University of Hawaii at Manoa, Honolulu, Hawaii, United States \\ 4 Urban Nature Research Center \& Section of Herpetology, Natural History Musem of Los Angeles County, Los Angeles, California, United States of \\ America \\ 5 Department of Ecology and Evolutionary Biology, Cornell University, Ithaca, New York, United States \\ 6 Biology Department, Louisiana State University, Baton Rouge, Louisiana, United State of America \\ 7 Department of Ecology and Evolutionary Biology \& La Kretz Center for California Conservation Science, University of California, Los Angeles, Los \\ Angeles, California, United States \\ Corresponding Author: Max R Lambert \\ Email address: mrl24@berkeley.edu
}

The red-eared slider turtle (Trachemys scripta elegans; RES) is often considered one of the world's most invasive species. Results from laboratory and mesocosm experiments suggest that introduced RES outcompete native turtles for key ecological resources, but such experiments can overestimate the strength of competition. We report on the first field experiment with a wild turtle community, involving introduced RES and a declining native species of conservation concern, the western pond turtle (Emys marmorata; WPT). Using a before/after experimental design, we show that after removing most of an introduced RES population, the remaining RES dramatically shifted their spatial basking distribution in a manner consistent with strong intraspecific competition. WPT also altered their spatial basking distribution after the RES removal, but in ways inconsistent with strong interspecific competition. However, we documented reduced levels of WPT basking post-removal, which may reflect a behavioral shift attributable to the lower density of the turtle community. WPT body condition also increased after we removed RES, consistent with either indirect or direct competition between WPT and RES and providing the first evidence that RES can compete with a native turtle in the wild. We conclude that the negative impacts on WPT basking by RES in natural contexts are more limited than suggested by experiments with captive turtles, although wild WPT do appear to compete for food with introduced RES. Our results highlight the importance of manipulative field experiments when studying biological invasions, and the potential value of RES removal as a management strategy for WPT. 
1 Full Title: Experimental removal of introduced slider turtles offers new insight into competition

2 with a native, threatened turtle

3

4 Short Title: Turtle competition

5

6 Max R. Lambert ${ }^{1 *}$, Jennifer M. McKenzie ${ }^{2}$, Robyn M. Screen ${ }^{3}$, Adam G. Clause ${ }^{4}$, Benjamin J.

7 Johnson ${ }^{5}$, Genevieve G. Mount ${ }^{6}$, H. Bradley Shaffer 7 , and Gregory B. Pauly ${ }^{5}$

$9{ }^{1}$ Department of Environmental Science, Policy, and Management, UC Bereley, Berkeley,

10 California, United Sates of America.

$11{ }^{2}$ Department of Forestry and Natural Resources, University of Kentucky, Lexington, Kentucky,

12 United Sates of America.

$13{ }^{3}$ Department of Biology, University of Hawaii at Manoa, Honolulu, Hawaii, United Sates of

14 America.

$15{ }^{4}$ Urban Nature Research Center \& Section of Herpetology, Natural History Museum of Los

16 Angeles County, Los Angeles, California, United Sates of America.

$17{ }^{5}$ Department of Ecology and Evolutionary Biology, Cornell University, Ithaca, New York, 18 United Sates of America. 
$19{ }^{6}$ Biology Department, Louisiana State University, Baton Rouge, Louisiana, United Sates of

20 America.

$21 \quad{ }^{7}$ Department of Ecology and Evolutionary Biology \& La Kretz Center for California

22 Conservation Science, University of California, Los Angeles, Los Angeles, California, United

23 Sates of America.

24 *Correspondence author: email - lambert.mrm@gmail.com, Twitter - @MaxRLambert

35 Abstract: The red-eared slider turtle (Trachemys scripta elegans; RES) is often considered one

36 of the world's most invasive species. Results from laboratory and mesocosm experiments

37 suggest that introduced RES outcompete native turtles for key ecological resources, but such 
38

39

40

41

42

experiments can overestimate the strength of competition. We report on the first field experiment with a wild turtle community, involving introduced RES and a declining native species of conservation concern, the western pond turtle (Emys marmorata; WPT). Using a before/after experimental design, we show that after removing most of an introduced RES population, the remaining RES dramatically shifted their spatial basking distribution in a manner consistent with strong intraspecific competition. WPT also altered their spatial basking distribution after the RES removal, but in ways inconsistent with strong interspecific competition. However, we documented reduced levels of WPT basking post-removal, which may reflect a behavioral shift attributable to the lower density of the turtle community. WPT body condition also increased after we removed RES, consistent with either indirect or direct competition between WPT and RES and providing the first evidence that RES can compete with a native turtle in the wild. We conclude that the negative impacts on WPT basking by RES in natural contexts are more limited than suggested by experiments with captive turtles, although wild WPT do appear to compete for food with introduced RES. Our results highlight the importance of manipulative field experiments when studying biological invasions, and the potential value of RES removal as a management strategy for WPT.

\section{Introduction}

The International Union for Conservation of Nature (IUCN) has labeled the red-eared slider turtle (Trachemys scripta elegans; RES) one of the "world's worst invasive species" (Lowe et al. 2000). RES are native to the central United States - from West Virginia through Texas to eastern New Mexico and north into Illinois - but are now established throughout much of the country. RES are also globally distributed, having been released on every continent except Antarctica with robust populations in Europe and Asia. These introductions predominantly result 
61 from the release of unwanted pet turtles (Kraus 2009; Rhodin et al. 2017). Results from

62 laboratory and mesocosm experiments suggest that RES can outcompete native European and

63

64

eastern North American freshwater turtles for food and basking sites (Cadi and Joly 2003, 2004;

Polo-Cavia et al. 2010, 2011; Pearson et al. 2015). While such controlled experiments are

informative, they can also inflate the effects of competition compared to in situ field

manipulations (Skelly 2002; Winkler and Van Buskirk 2012). Comparing laboratory and

mesocosm experiments with field manipulations is a critical step to a more complete

understanding of the strength and mechanisms underlying species interactions in nature.

However, to our knowledge, no study has experimentally tested for competition between nonnative RES and any native turtle species in the wild.

Basking sites are a key resource for thermoregulation, disease control, and reproduction in freshwater turtles (Ernst and Lovich 2009), and previous ex situ experiments suggest that basking sites are an important axis of competition between native turtles and introduced RES (Cadi and Joly 2003; Polo-Cavia et al. 2010). Prior work in the University of California, Davis Arboretum waterway (hereafter, UCD Arboretum) found that introduced RES and native western pond turtles (Emys marmorata; WPT) sometimes bask at the same sites (Fig 1), although they tend to use basking sites that differ physically and spatially (Lambert et al. 2013). In particular, WPT and RES predominantly bask in the western and eastern ends of the UCD Arboretum, respectively. While WPT basking is not related to particular habitat characteristics, RES basking is related to sites with more human activity, steel mesh basking substrates, deeper water, and shallower slopes. Whether these basking site differences are the result of species-specific habitat choices or competition has never been resolved and requires an experimental approach. 
84

85

and consume a broad array of food items, though they tend to shift from higher rates of carnivory when young to higher rates of herbivory as adults (Ernst and Lovich 2009). Even so, laboratory and mesocosm experiments suggest RES might directly interfere with native turtle food consumption through aggressive behaviors or higher food consumption rates, which can limit food availability and growth rates of less competitive turtles (Cadi and Joly 2004; Polo-Cavia et al. 2011; Pearson et al. 2015). Additionally, if turtle densities are high for a given habitat, exploitative competition could limit food availability, both intra- and interspecifically, and therefore decrease growth rates and / or body condition of native species.

Here, we present the results of an in situ field experiment where we substantially reduced the introduced RES population at the UCD Arboretum to test for competition with WPT. Because the waterway is disjunct from other turtle populations, the UCD Arboretum represents a closed system for WPT that is well suited for experimental manipulations; natural immigration/emigration is not possible for freshwater turtles in this system, although occasional human-assisted transport does occur, particularly with RES released into the waterway. Our experiment is the first to explicitly test whether invasive species removal, a commonlyadvocated management practice for invasive species including RES (Gaeta et al. 2015; GarcíaDíaz et al. 2017), influences the basking behavior and body condition of a native turtle in the wild. If the distribution of WPT basking is a result of direct, competitive exclusion by RES from optimal basking sites, then RES removal should result in an increase of post-removal WPT basking at sites previously dominated by RES. Alternatively, if WPT basking activity does not significantly change in this manner after RES removal, then existing behavioral basking differences between the two species likely reflect species-specific habitat preferences, 
106 competitive superiority of WPT, or both. We also assessed WPT body condition pre- and post-

107 removal as a proxy for whether removing RES improves WPT access to food resources. If

108 introduced RES compete with WPT for food, then removing RES should result in an increase in

109 WPT body condition. Given the broad overlap of these two species across California (Thomson

110 et al. 2010, 2016), the range-wide imperilment of WPT (Spinks et al. 2003; Thomson et al.

111 2016), and the current Status Review for possible WPT listing under the U.S. Endangered

112 Species Act (USFWS 2015), this experiment is directly relevant to ongoing WPT management 113 actions.

\section{Methods}

116 UC Davis IACUC Protocols \#15263 and \#16227 and California Department of Fish and Wildlife

117 Scientific Collecting Permits \#2480, \#4307, and \#11663 approved this work. We conducted all 118 analyses in the $\mathrm{R}$ statistical language (version 3.5.2).

Study Site

121 Our study took place at the UCD Arboretum (38.53, -121.76), a permanent waterway extending

122 along the southern border of the UC Davis campus, Yolo County, California, USA (Fig 1). The

123 UCD Arboretum was formed in the 1870s when the historical north fork channel of Putah Creek

124 was diverted into the south fork (Larkey 1980). This waterway is $2.4 \mathrm{~km}$ long, ca. 4 ha in surface 125 area, and averages $15 \mathrm{~m}$ wide and $1 \mathrm{~m}$ deep (Spinks et al. 2003). Terrestrial habitat surrounding 126 the waterway is irrigated and landscaped with predominantly non-native vegetation (Spinks et al. 127 2003). A 1.5-2.5 m wide paved path encircles the entire waterway within 5-10 $\mathrm{m}$ of the water's 128 edge. This path is regularly used by pedestrians, bicyclists, and maintenance vehicles which 
129 influence turtle basking (Lambert et al. 2013, Costa 2014). The waterway's shoreline_including

130 basking sites - is a combination of concrete, exposed dirt, and landscaping steel mesh which has

131 been exposed by erosion (Lambert et al. 2013).

132

133 Turtle trapping and RES removal

134 Across the UCD Arboretum, we deployed baited submersible traps in optimal habitat for both 135 RES and WPT over approximately 900 trap-days from 10 July-1 August, 2011 and again from

136 13-29 September, 2011. We supplemented this trapping with dip netting and opportunistic hand 137 captures during both periods, and with a fyke net and a basking trap during the latter period. Dip 138 netting and hand captures were targeted at RES but other trapping was not. We removed and 139 euthanized all RES, depositing most specimens at the UC Davis School of Veterinary Medicine, 140 the Natural History Museum of Los Angeles County, or the UC Davis Museum of Wildlife and 141 Fish Biology. Beginning in the 1996, our group uniquely marked each captured WPT with scute 142 notches using a handheld file or (juveniles only) nail clippers (Spinks et al. 2003). We similarly 143 marked any new WPT during this trapping effort. We used linear regression to test whether our 144 trapping depleted the RES population over time by regressing cumulative RES captures against 145 trapping day for adult RES (Krebs 1989). Using likelihood ratio tests (Crawley 2013) to assess 146 model fits, we compared a quadratic model, which would indicate population depletion, to a 147 linear model, which would indicate that the RES population was not leveling off with our 148 removal effort. 149 150 WPT Body Condition 
151 To estimate changes in WPT body condition, we trapped for one week the year following RES

152 removal, from 27 May-2 June, 2012. Due to logistical constraints we were unable to trap later in

153 the summer at a similar time as in 2011. Differences in trapping dates may influence body

154 condition analyses because females may be gravid and therefore heavier in the earlier 2012

155 sampling or because all turtles may have had more time to put on mass during the later 2011

156 sampling. However, these effects are likely limited. In both 2011 and 2012 we measured WPT

157 plastron length (notch-to-notch; mm) with dial calipers and body mass (g) with Ohaus CS2000

158 digital pan scales (Iverson and Lewis 2018). We used a linear mixed-effects model (function

159 'Imer', R package "Ime4"; Bates et al. 2015) to test whether WPT body condition (i.e.,

160 differences in mass controlling for body length) changed after the RES removal (Cadi and Joly

161 2003; Schulte-Hostedde et al. 2005; Litzgus et al. 2008). Our model of WPT mass controlled for

162 plastron length and included treatment (pre- or post-removal) and sex as fixed effects and

163 individual WPT as a random effect to control for repeated measures. This model simultaneously

164 regresses body mass against plastron length and tests for differences in the residuals of this

165 model between study year and sex. We used likelihood ratio tests to assess the significance $(\alpha<$

166 0.05) of fixed effects and removed non-significant variables from our model. We obtained full

167 model conditional $\mathrm{R}^{2}\left(\mathrm{cR}^{2}\right)$ for fixed and random effects combined and a marginal $\mathrm{R}^{2}\left(\mathrm{mR}^{2}\right)$ for

168 the model's fixed effects alone (function 'r.squaredGLMM', package "MuMIn”, Barton 2018).

169

\section{Basking Site Monitoring}

171 We conducted binocular surveys of 24 pre-selected basking sites (Fig 1) for 34 total days-16

172 days pre-removal between 18 March and 22 April 2010 (Lambert et al. 2013) and 18 days post-

173 removal between 18 March and 22 April 2012. Following Lambert et al. (2013), we performed 
174 all surveys between 1000 and $1500 \mathrm{hr}$ to coincide with the expected maximum turtle basking

175 activity during this time of year. We surveyed all sites once daily in rapid succession to avoid

176 counting the same turtle at multiple sites. Each survey was performed in under one hour, at a

177 distance of ca. 10-100 m from turtles, and did not noticeably disturb basking turtles. MRL and S.

178 Nielsen conducted basking surveys in 2011 and JMM and RMS conducted 2012 surveys; all

179 surveyors were trained by GBP and HBS. During each survey we recorded the number of

180 individuals of each species basking at each basking site as well as water temperature because we

181 previously found that basking activity of both species increases more with warmer water

182 temperatures than with air temperatures (Lambert et al. 2013). We also obtained air temperature

183 data from the UC Davis Russell Ranch Weather Station which is located ca. $4 \mathrm{~km} \mathrm{NW}$ of the

184 UCD Arboretum.

Modeling the Effects of RES Removal on Turtle Basking

187 We tested for changes in the relative basking distribution of WPT and RES (i.e., the ratio of WPT:RES) across the UCD Arboretum pre- and post-RES removal using a generalized linear mixed effects model (GLMM) with a binomial family for proportion data (function 'glmer', R package "lme4"). A binomial GLMM accounts for binary data (two species here) and variation in sample sizes across basking sites and survey days. We modeled WPT:RES basking as a function of treatment (pre- or post-removal) and the distance of each basking site from the west end of the UCD Arboretum because turtle basking distributions were previously shown to vary west-east (Lambert et al. 2013). We accounted for repeated measures by treating survey date as a random effect (Lambert et al. 2013). To explore site-specific changes in the ratio of the two

196 species, we also used individual binomial GLMMs for each basking site. 
In addition, we modeled the absolute basking abundance of both species pre- and post-

198

199

200

201

202

203

204

205

206

207

208

209

210

211

212

213

214

215

216

217

218 removal using Poisson GLMMs (function 'glmer', R package “Ime4”) for count data. Our approach here was the same as with the binomial GLMM and, if an interaction between treatment and distance from the west end was significant, we used individual GLMMs for each year to test the pattern and strength of turtle basking distributions across the UCD Arboretum in each year. To test whether certain basking sites made up larger or smaller proportions of total WPT basking observations pre- or post-removal, we used contingency tables, focusing on the five most heavily-used turtle basking sites (combined for both species) pre-removal (sites $\mathrm{P}, \mathrm{O}$, E, Q, and R) and site X, the most heavily-used turtle basking site post-removal.

Results:

Trapping and RES Removal

We removed and euthanized 177 RES (100.6 kg total biomass), including 28 adult males (16.3 $\mathrm{kg}), 72$ adult females $(79.4 \mathrm{~kg})$, and 77 juveniles $(4.9 \mathrm{~kg}$, defined as $\leq 100 \mathrm{~mm}$ carapace length; Ernst and Lovich 2009). A quadratic (rather than linear) model fit our data best (likelihood ratio test $\mathrm{p}<0.0001$, full model $\mathrm{R}^{2}=0.95$ ) and showed RES captures leveling off, signifying we had removed a substantial fraction of the RES population.

We also captured, marked, and released 115 unique WPT (62.7 kg total biomass) comprising 51 males $(36.1 \mathrm{~kg}), 36$ females $(24.1 \mathrm{~kg})$, and 28 juveniles $(2.5 \mathrm{~kg}$, defined as $\leq 110$ mm plastron length; Holland, 1991).

WPT Body Condition 
219 While we trapped a larger number of WPT in each year, we trapped 25 unique adult WPT in 220 both 2011 and 2012; we used these 25 WPT for the body condition analysis. The body condition

221 linear model showed no interaction between treatment and sex $(p=0.92)$ and so we removed this

222 interaction from the model. Sex $(\mathrm{p}=0.009)$, treatment $(\mathrm{p}<0.001)$, and plastron length $(\mathrm{p}<$

2230.0001 ) were significant (full model $\mathrm{cR}^{2}=0.95, \mathrm{mR}^{2}=0.86$ ). For a given plastron length, males

224 were on average $61.54 \mathrm{~g}( \pm 23.55 \mathrm{~g})$ heavier than females. Although the $25 \mathrm{WPT}$ measured

225 before and after RES removal showed individual variation in their degree of body condition

226 change post-removal (Fig 2A), on average they were $39.80 \mathrm{~g}( \pm 9.92 \mathrm{~g})$ heavier for a given

227 plastron length post-removal (Fig 2B).

228

229 Basking Site Monitoring

230 We recorded 283 WPT and 645 RES observations in 2010 but only 43 WPT and 61 RES

231 observations in 2012. Although the reduction in numbers of observed WPT was unexpected, we 232 do not believe this reflects a decline in the WPT population. From 27 May-2 June 2012, we

233 trapped 54 unique WPT over seven days and a Schnabel multiple capture-mark-recapture

234 population estimate (Krebs 1989) derived from trapping data (beginning in the mid 2000s)

235 suggest that ca. 162 WPT (including 10 newly-marked juveniles) were present in the UCD

236 Arboretum immediately after our post-removal surveys (McKenzie, Screen, and Pauly unpubl.);

237 this estimate is similar to pre-removal estimates of WPT population size (ca.146 WPT, including

23818 newly-marked juveniles). Given these estimates, we are confident that the WPT population

239 was essentially unchanged during our experiment, and thus our focus on the relative basking

240 distributions of turtles at monitored basking sites meaningfully reflects the impact of our removal

241 experiment and not a catastrophic decline in WPT.

Peer] reviewing PDF | (2019:04:36973:1:1:NEW 29 Jun 2019) 
243 sites used post-removal (Fig 3A, B). We recorded WPT basking at 15 of 24 basking sites pre-

244 removal, but at only 8 of 24 sites post-removal. WPT were absent from 8 sites they used pre-

245 removal (although of these, only 2 were frequently used pre-removal: sites A and N) and were

246 present at one additional site where they were not recorded pre-removal (site B). We recorded

247 RES basking at 17 of 24 basking sites pre-removal, and only 8 of 24 sites post-removal (Fig 3A,

248 B). RES were absent from 9 sites they used pre-removal and were not recorded using new sites

249 post-removal.

250

Water temperatures were warmer in $2010(17.0 \mathrm{C} \pm 0.24 \mathrm{SE})$ than in $2012(15.4 \mathrm{C} \pm 0.36$

251

252

253

254

255

256

257

258

259

260

261

262

Effects of RES Removal on Turtle Basking 
263 The interaction between removal treatment and distance from the west end of the UCD

264 Arboretum was not significant $(p=0.18)$ and was removed from the model. Both treatment $(p<$ $2650.0001)$ and distance from the west end $(\mathrm{p}<0.0001)$ were retained $\left(\mathrm{cR}^{2}=0.31, \mathrm{mR}^{2}=0.31\right)$.

266 The non-significant interaction indicates the removal did not change the ratio of

267 WPT:RES basking distributions across the UCD Arboretum. Both pre- and post-removal, the

268 basking distribution of turtles was WPT-biased in the west end and RES-biased in the east end

269 (Fig 4). However, the proportion of basking individuals that were WPT increased from 30.5\%

270 pre-removal to $41.3 \%$ post-removal ( $\mathrm{p}<0.0001$; Tukey's post-hoc test, function 'glht', package

271 "multcomp"). Individual binomial GLMMs for each basking site showed removal treatment

272 effects on the WPT:RES basking ratio for site Q $(\mathrm{p}=0.002,9 \% \mathrm{WPT}$ to 55\% WPT; Fig 3) and a

273 marginal effect for site O $(\mathrm{p}=0.09,30 \%$ WPT to 75\% WPT; Fig 3$)$. All other individual basking

274 sites showed no differences (all $\mathrm{p}>0.1)$.

275 Pre-removal, the WPT basking distribution declined from west to east, and post-removal

276 WPT basking had a relatively flat distribution (Fig 5). We detected a shift in the absolute basking

277 distribution of WPT with a significant interaction between removal treatment and distance from

278 the west end (Poisson GLMM, $\mathrm{p}=0.012, \mathrm{cR}^{2}=0.23, \mathrm{mR}^{2}=0.06$ ). Individual GLMMs for each

279 year indicated that distance from the west end was significantly associated with WPT basking

280 abundance in the pre-removal year $\left(\mathrm{p}<0.012, \mathrm{cR}^{2}=0.27, \mathrm{mR}^{2}=0.03\right)$ but not in the post-

281 removal year $(\mathrm{p}=0.55)$.

282 WPT predominantly used the same basking sites post-removal but showed a more even

283 distribution across basking sites, with more basking activity at two center-east sites (Q and X)

284 compared to before the RES removal (Fig 3A, B). Contingency table analyses showed that sites

$285 \mathrm{Q}(\mathrm{p}=0.01)$ and $\mathrm{X}(\mathrm{p}=0.001)$ encompassed larger proportions of total WPT basking 
286 observations post-removal than pre-removal (Fig 3A, B). All other sites made up similar

287 proportions pre- and post-removal (all $\mathrm{p}>0.1$ ), though some sites had generally low basking

288 activity (Fig 3A, B), possibly limiting our power to detect shifts.

WPT. Even so, if more eastern sites that were dominated by RES pre-removal (e.g., sites O, P, these sites post-removal. We did not see this shift.

After removal, remaining RES were sparse throughout much of the UCD Arboretum and concentrated in the east end (Fig 3B, 5). For RES, a Poisson GLMM indicated a significant interaction between treatment and distance to the west end $\left(\mathrm{p}<0.0001, \mathrm{cR}^{2}=0.30, \mathrm{mR}^{2}=0.16\right)$. Individual GLMMs for each year showed a positive relationship between RES basking and the distance to the west end pre-removal $\left(\mathrm{p}<0.0001, \mathrm{cR}^{2}=0.27, \mathrm{mR}^{2}=0.02\right)$ and post-removal $(\mathrm{p}<$ $\left.0.0001, \mathrm{cR}^{2}=0.14, \mathrm{mR}^{2}=0.14\right)$. The distance of each basking site from the west end explained substantially more of the variation in RES basking abundance post-removal than pre-removal, indicating that remaining RES concentrated in the east end more strongly after we removed most of the RES population (Fig 3B, 5). Contingency table analyses indicated that sites $\mathrm{E}, \mathrm{O}, \mathrm{P}$, and R (Fig 3) comprised lower proportions of total RES basking observations after the removal and site $\mathrm{X}$ (Fig 3) comprised a higher proportion (all $\mathrm{p}<0.05)$. Site Q made up similar proportions of total RES observations in both years $(\mathrm{p}=0.14)$. used heavily (particularly sites $\mathrm{O}$ and P; Fig 3) and shifted towards the east end of the UCD and that, prior to our experiment, RES densities were high enough for intraspecific competition 
309 to force many RES into less preferred areas of the waterway. Our previous work showed that

310 RES basking activity was highest at sites with shallow slopes, deeper water adjacent to the site, a

311 steel mesh (rather than concrete or dirt) substrate, and high human activity (Lambert et al. 2013).

312 Post-removal, RES basking activity was highest at the two sites (V and X) that maximized this

313 combination of variables based on 2010 surveys (Fig 4B from Lambert et al. 2013).

\section{Discussion}

Our experimental removal dramatically altered both RES and total turtle density in the

317 UCD Arboretum by eliminating over half of the turtles in the waterway. Given the high

population density of RES and given that we likely removed the majority of the RES population, we consider our RES removal effort substantial enough to have exerted an effect on WPT if the two species compete for food or basking sites. Our removal experiment offers new insights into competition for basking habitats and food between introduced RES and native WPT, producing four key results.

First, the prevalence of basking turtles at our survey sites post-removal was about $15 \%$ of that pre-removal, and this reduction in basking observations was measured in both species. We have no evidence that the removal of RES negatively affected the WPT population size, and a follow-up trapping survey confirmed that the number of WPT present remained roughly constant. Rather, it appears that the overall lower density of turtles in the UCD Arboretum allowed many WPT to either shift their basking activity patterns, redistribute themselves to sites that we were not monitoring, or both. Environmental differences, including cooler water temperatures during our post-removal monitoring, may also explain the lower WPT basking 
332 during our basking surveys would support maximal WPT basking activity (Lambert et al. 2013).

333 It seems unlikely that differences in observers between years would have impacted these

334 findings.

Second, after removing RES, we found that WPT basking activity at our monitoring sites shifted but did not increase at sites previously dominated by RES. Thus, we did not find evidence of strong interspecific competition for those sites. Interspecific competition is greatest at higher densities and the effects of an introduced competitor can similarly manifest or become most pronounced when the introduced species is at high densities (Gurnell et al. 2004). Therefore, competition is presumably greatest at high densities of RES (or turtles generally) and perhaps influenced by the relative densities of both species. While earlier laboratory and mesocosm experiments suggest introduced RES outcompete native turtles for basking sites and other resources (Cadi and Joly 2003; Polo-Cavia et al. 2010; Pearson et al. 2015), our results suggest more subtle effects found in complex, natural communities that are poorly predicted by simplified mesocosm experiments (Skelly 2002, Winkler and Van Buskirk 2012). and X) consistent with their previously identified preferred habitat characteristics (Lambert et al. 2013), suggesting that high RES densities prior to our experimental removal produced strong intraspecific competition, forcing many RES to use less-preferred basking habitat.

Fourth, we found that removing RES led to an increase in WPT body condition, suggesting that these turtle species compete for food. Whether this reflects interference competition (direct interactions between the two species), exploitation competition (both species indirectly competing for overlapping food resources), or a combination of the two is unclear.

354 Experimental work on RES and other native turtles suggests RES may behaviorally prevent 
355 native turtles from obtaining sufficient food (Cadi and Joly 2004, Polo-Cavia et al. 2011, Pearson

356 et al. 2015), and our experimental removal may have reduced such interference if it does exist in

357 this population. However, we also removed a substantial portion of the overall turtle community

358 thereby reducing the overall pressure on food resources in the system. While differences in

359 trapping dates between the two years (earlier post-removal) may have influenced our results, we

360 think such effects are limited. The absence of an interaction between sex and treatment indicates

361 that male and female WPT responded similarly to RES removal and suggests that differences in

362 trapping date did not influence our results because of gravid females. Additionally, later trapping

363 pre-removal could have allowed WPT to gain more mass over the active season compared to

364 earlier trapping post-removal, making it hypothetically more challenging to detect a positive

365 effect of RES removal on WPT body condition. Because of this, our body condition results may

366 be a conservative estimate of body condition increase. Regardless of the mechanism, the ca. $40 \mathrm{~g}$

367 average increase in body condition we detected is substantial given that all WPT in our analysis

368 pre-removal weighed under 1,100 g. To our knowledge, this result represents the first evidence

369 from wild populations that introduced RES compete with native turtles for food and that RES

370 removal can lead to improved body condition of native turtles.

371

372

Should We Remove RES to Benefit Declining Native Turtles?

373 A recent summary of research goals for effective conservation of WPT (Thomson et al. 2016)

374 identified the need for a clearer quantitative understanding of the impact of introduced RES.

375 Controlling invasive species is a substantial commitment that rarely eliminates the entire

376 population, particularly in situations with continual introductions (Kikillus et al. 2012, Gaeta et

377 al. 2015, Garcia-Diaz et al. 2017). Removing 177 RES from the UCD Arboretum was an 
378 intensive effort requiring $>2,000$ person-hours of field work across 40 days. A similar level of

379 effort would conservatively cost the California Department of Fish and Wildlife \$26,000-

$380 \$ 31,000$ in Scientific Aid hourly wages (L. Patterson, pers. comm.). While our study suggests

381 that removing RES does influence native turtle basking ecology and feeding, the potential

382 benefits with respect to short-term basking-site usage appear quantitatively modest. However,

383 the substantial increase in WPT body condition during the year following the RES removal

384 suggests that removing RES meaningfully increased resource availability for WPT. Whether

385 these returns justify the effort may well depend on several variables, including RES abundance /

386 density, attitudes of local human residents to introduced RES, disease risk (Héritier et al. 2017),

387 other potential axes of competition (e.g., nesting sites), and additional aspects of ecosystem

388 health.

Our results also provide evidence that RES introductions may affect native turtles simply

by inflating turtle densities in general (regardless of species identity). Therefore, removing RES

may not necessarily relieve native turtles from a dominant competitor but, rather, may relieve

ecological or behavioral pressures associated with high turtle densities and could conceivably

result in unexpected responses by native species. One such unexpected response here was the

substantial decrease in overall WPT basking observations after we removed over half of the

turtle community, a result that suggests a change in WPT behavior and habitat use that our

experimental design, with fixed monitoring sites, failed to capture. Unlike many other freshwater

turtles, WPT are aggressive baskers - threatening, biting, pushing, and ramming other turtles

from basking sites — and prefer to bask alone or in low numbers (Bury and Wolfheim 1973).

Reduced turtle densities post-removal may thus have allowed WPT to occupy other basking 
401 removal was likely influenced by there simply being fewer turtles overall competing for food in

402 the UCD Arboretum. Improved body condition may also be the result of WPT adopting preferred

403 basking behaviors, thereby improving digestive efficiency and mass gain. Future studies,

404 including dietary research, that include unmanipulated control sites, pre-removal surveys that

405 span multiple years and account for year-to-year variation, as well as a design that tracks the

406 behavior of native turtles pre- and post-RES removal (e.g., using GPS-enabled radio

407 transmitters) may better elucidate these unexpected outcomes on native turtles. Overall, our

408 analyses suggest WPT responded to RES removal in a manner consistent with interspecific

409 competition for food but inconsistent with strong interspecific competition for basking habitats,

410 implying that removing RES may well be an important management strategy in some situations.

Alternatively, the direct management of basking habitat may be a more generally

412

413

414

415

416

417

418

419

420

421

tractable conservation activity for WPT (Spinks et al. 2003, Thomson et al. 2016). In human-

modified waterways, removal of floating basking sites for flood control and aesthetics (Spinks et

al., 2003) could exacerbate competition for basking sites. Emerging research suggests that

experimentally-added floating logs are preferred by WPT compared to bank-side basking sites

and are more heavily used by WPT than RES, especially when they are isolated from human

activities (Cossman et al. unpubl.). Adding artificial basking sites that favor WPT, alone or in

combination with RES population reduction, is a simple, comparatively inexpensive

manipulation that should be explored in future field experiments. 
422 The primary limitations of our study center on interpreting our basking results. We expected to

423 observe fewer basking RES in the second year of study due to our intense removal effort but did

424 not expect a concomitant decline in WPT observations. It is possible that water temperature,

425 other environmental variation, or unforeseen consequences of our manipulation resulted in

426 reduced overall turtle basking activity, or (more likely to us) radical shifts in basking to new and

427 unmonitored locations, after the RES removal. Unfortunately, we cannot confidently identify

428 which factor(s) resulted in fewer WPT basking observations. Although we studied both basking

429 and feeding, we also recognize that our experiment did not address other potentially important

430 axes of competition that are important for the continued recruitment and persistence of WPT

431 populations. While we employed a before-after comparative design, the use of unmanipulated

432 control sites would have improved our ability to make stronger inferences in this study. We do

433 not believe that the lower number of WPT basking observations confounds our results because

434 our analyses of relative basking distribution differences between species and years can

435 accommodate sample size differences. Additionally, our analyses found that residual RES shifted

436 their basking in intuitive ways (i.e., towards sites with preferred characteristics), increasing

437 confidence in our results. While field experiments offer more biological realism than

438 experiments in captivity, that added complexity may also yield unexpected results, such as

439 changing a focal species' behaviors or habitat use.

\section{Conclusions}

442 We present the first in situ field manipulation testing for competition between non-native RES

443 and native turtles. Consistent with expectations based on laboratory and mesocosm studies, RES

444 removal increased WPT body condition and altered WPT basking activity. However, contrary to 
445 expectations, this change in basking was not consistent with strong competition between RES

446 and WPT for individual basking sites in the UCD Arboretum. Our results offer evidence for

447 intraspecific competition for food and basking sites at high RES densities, underscore the value

448 of manipulative field experiments in studying biological invasions, and suggest that removing

449 introduced RES could be considered a useful, albeit logistically challenging, tool for managing

450 wild WPT in some contexts. We encourage other researchers to replicate our field-based

451 experiment, perhaps using control sites or multiple years of pre-removal observations. These

452 modifications to our protocol would improve the ability to interpret competition between RES

453 and native turtles and the magnitude of behavioral shifts that occur when removals lead to

454 changes in both relative and absolute turtle densities.

Data Availability: Basking and body size data are available in the electronic supplementary

457 material.

458 Acknowledgments: This work began while all authors were at UC Davis. The UCD

Arboretum staff, members of the Peter Moyle and Janet Foley labs, Nicholas Buckmaster,

460 Lauren Cassidy, Jillian Howard, Anna Jordan, Brian Mahardja, Hilary Rollins, Bryce Sullivan, 461 and Matthew Young provided invaluable assistance.

\section{References}

Barton, K. 2018. Package ‘MuMIn’. MuMIn: Multi-Model Inference. R package version 1.42.1. https://CRAN.R-project.org/package=MuMIn 
using lme4. Journal of Statistical Software. 67: 1-48. doi:10.18637/jss.v067.i01.

469 Bury, R. B. and J. H. Wolfheim. 1973. Aggression in free-living pond turtles (Clemmys marmorata). BioScience 23: 659-662.

471

472

473

474

475

476

477

478

479

480

481

482

483

484

485

486

487

Cadi, A. and P. Joly. 2003. Competition for basking places between the endangered European pond turtle (Emys orbicularis galloitalica) and the introduced red-eared slider (Trachemys scripta elegans). Canadian Journal of Zoology 81: 1392-1398.

Cadi, A. and P. Joly. 2004. Impact of the introduction of the red-eared slider (Trachemys scripta elegans) on survival rates of the European pond turtle (Emys orbicularis). Biodiversity and Conservation 13: 2511-2518.

Costa, Z. J. 2014. Responses to predators differ between native and invasive freshwater turtles: environmental context and its implications for competition. Ethology 120: 633-640.

Crawley, M. J. 2013. The R Book. Wiley \& Sons, Chichester, West Sussex, UK.

Ernst, C. H. and J. E. Lovich. 2009. Turtles of the United States and Canada. Johns Hopkins University Press, $2^{\text {nd }}$ Ed. 840 pages.

Gaeta, J. W., Hrabik, T. R., Sass, G. G., Roth, B. M., Gilber, S. J., and M. J. Vander Zanden. 2015. A whole-lake experiment to control invasive rainbow smelt (Actinopterygii, Osmeridae) via overharvest and a food web manipulation. Hydrobiologia 746: 433-444.

García-Díaz, P., Ramsey, D. S. L., Woolnough, A. P., Franch, M., Llorente, G. A., Montori, A., Buenetxea, X., Larrinaga, A. R., Lasceve, M., Álvarez, A., Traverso, J. M., Valdeón, A., Crespo, A., Rada, V., Ayllón, E., Sancho, V., Lacomba, J. I., Bataller, J. V., and M. 
Lizana. 2017. Challenges in confirming eradication success of invasive red-eared sliders. Biological Invasions 19: 2739-2750.

490

491

492

493

494

495

496

497

498

499

500

501

502

503

504

505

506

507

Gurnell, J., Wauters, L. A., Lurz, P. W. W., and G. Tosi. 2004. Alien species and interspecific competition: effects of introduced eastern grey squirrels on red squirrel population dynamics. Journal of Animal Ecology 73: 26-35.

Héritier, L., A. Valdeón, A. Sadaoui, T. Gendre, S. Ficheux, S. Bouamer, N. Kechemir-Issad, L. Du Preez, C. Palacios, and O. Verneau. 2017. Introduction and invasion of the red-eared slider and its parasites in freshwater ecosystems of southern Europe: Risk assessment for the European pond turtle in wild environments. Biodiversity and Conservation 26: $1817-$ 1843.

Holland, D. C. 1991. A Synopsis of the Ecology and Status of the Western Pond Turtle Clemmys marmorata in 1991. Report to National Ecological Research Center. United States Fish and Wildlife Service, San Simeon, CA.

Iverson, J. B. and E. L. Lewis. 2018. How to measure a turtle. Herpetological Review 49: 453460.

Kikillus, K. H., Hare, K. M., and S. Hartley. 2012. Online trading tools as a method of estimating propagule pressure via the pet-release pathway. Biological Invasions 14: 2657-2664.

Kraus, F. 2009. Alien Reptiles and Amphibians, a Scientific Compendium and Analysis. Springer, Netherland. 563 pp.

Krebs, C. J. 1989. Ecological Methodology. Menlo Park, CA, USA, 765 pp.

Peer) reviewing PDF | (2019:04:36973:1:1:NEW 29 Jun 2019) 
508 Lambert, M. R., Nielsen, S. N., Wright, A. N., Thomson, R. C., and H. B. Shaffer. 2013. Habitat

509 features determine the basking distribution of introduced red-eared sliders and native western pond turtles. Chelonian Conservation and Biology 12: 192-199.

511

512

513

514

515

516

517

518

519

520

521

522

523

524

525

526

527

528

Larkey, J. L. 1980. Davisville '68 The History and Heritage of the City of Davis, Yolo County, California. Davis, CA: Davis Historical and Landmarks Commission, 240 pp

Litzgus, J. D., Bolton, F., and A. I. Schulte-Hostedde. 2008. Reproductive output depends on body composition in spotted turtles (Clemmys guttata). Copeia 2008: 86-92.

Lowe, S., Browne, M., Boudjelas, S., and M. De Poorter. 2000. 100 of the World's Worst Invasive Alien Species. A Selection from the Global Invasive Species Database. The Invasive Species Specialist Group (ISSG) of the Species Survival Commission (SSC) of the World Conservation Union (IUCN), $12 \mathrm{pp}$.

Pearson, S. H., Avery, H. W., and J. R. Spotila. 2015. Juvenile invasive red-eared slider turtles negatively impact the growth of native turtles: implications for global freshwater turtle populations. Biological Conservation 186: 115-121.

Polo-Cavia, N., Lopez, P., and J. Martin. 2008. Interspecific differences in responses to predation risk may confer competitive advantages to invasive freshwater turtle species. Ethology 114: $115-123$.

Polo-Cavia, N., Lopez, P., and J. Martin. 2010. Competitive interactions during basking between native and invasive freshwater turtle species. Biological Invasions 12: 2141-2152.

Polo-Cavia, N., Lopez, P., and J. Martin. 2011. Aggressive interactions during feeding between native and invasive freshwater turtles. Biological Invasions 13: 1387-1396. 
529 Rhodin, A. G. J., Iverson, J. B., Bour, R., Fritz, U., Georges, A., Shaffer, H. B., and P. P. van

530 Dijk. 2017. Turtles of the World. Annotated checklist and atlas of taxonomy, synonymy, 531 distribution, and conservation status ( $8^{\text {th }}$ Ed.). Chelonian Research Monographs 7: 1-292.

532 Schulte-Hostedde, A. I., Zinner, B., Millar, J. S., and G. J. Hickling. 2005. Restitution of mass533 size residuals: validating body condition indices. Ecology 86: 155-163.

534 Skelly, D. K. 2002. Experimental venue and estimation of interaction strength. Ecology 83: $535 \quad 2097-2101$.

536

537

538

539

540

541

542

543

544

545

546

547

548

Spinks, P. Q., Pauly, G. B., Crayon, J. J., and H. B. Shaffer. 2003. Survival of the western pond turtle (Emys marmorata) in an urban California environment. Biological Conservation 113: $257-267$.

Thomson, R. C., Spinks, P. Q., and H. B. Shaffer. 2010. Distribution and abundance of invasive red-eared sliders (Trachemys scripta elegans) in California's Sacramento River basin and possible impacts on native western pond turtles (Emys marmorata). Chelonian Conservation and Biology 9: 297-302.

Thomson, R. C., Wright, A. N., and H. B. Shaffer. 2016. California Amphibian and Reptile Species of Special Concern. University of California Press, Oakland, CA. 408 pp.

United States Fish \& Wildlife Service. 2015. Endangered and threatened wildlife and plants; 90day findings on 10 petitions. Federal Register 80: 19259-19263.

Winkler, J. D. and J. Van Buskirk. 2012. Influence of experimental venue on phenotype: multiple traits reveal multiple answers. Functional Ecology 26: 513-521. 


\section{Figure 1}

The UC Davis Arboretum waterway, turtle basking sites, and basking turtles.

Map (A) of the UC Davis Arboretum outlined with a dashed white line with the waterway in blue and turtle basking sites displayed as white-rimmed red circles. Seen basking (B) are a native western pond turtle and an introduced red-eared slider side-by-side in the Arboretum. Map data @ Bing 2019. Photo credit Max Lambert.

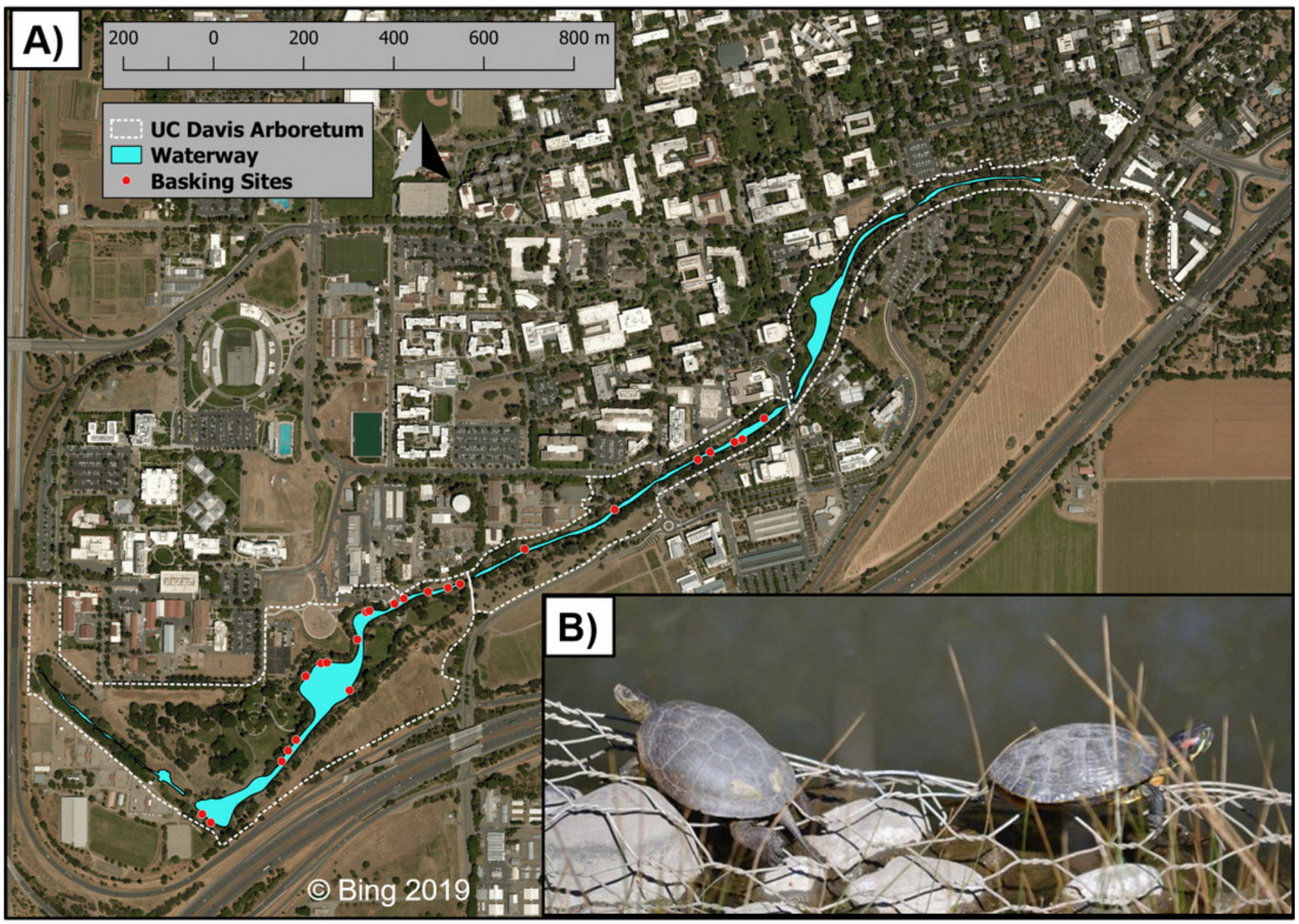


Figure 2

Native western pond turtle (WPT) body condition before and after introduced red-eared slider (RES) removal.

Body condition is shown as the residuals of body mass regressed against plastron length. Individual WPT varied in their body condition response to introduced RES removal (A) but body condition generally improved. On average (B) WPT are $39.80 \mathrm{~g}$ heavier after RES removal. Boxplot hinges show the $25^{\text {th }}$ and $75^{\text {th }}$ body condition percentiles, whiskers show the extent of data within 1.5 times the interquartile range, and the center line is the median for each treatment year pre- and post-removal.

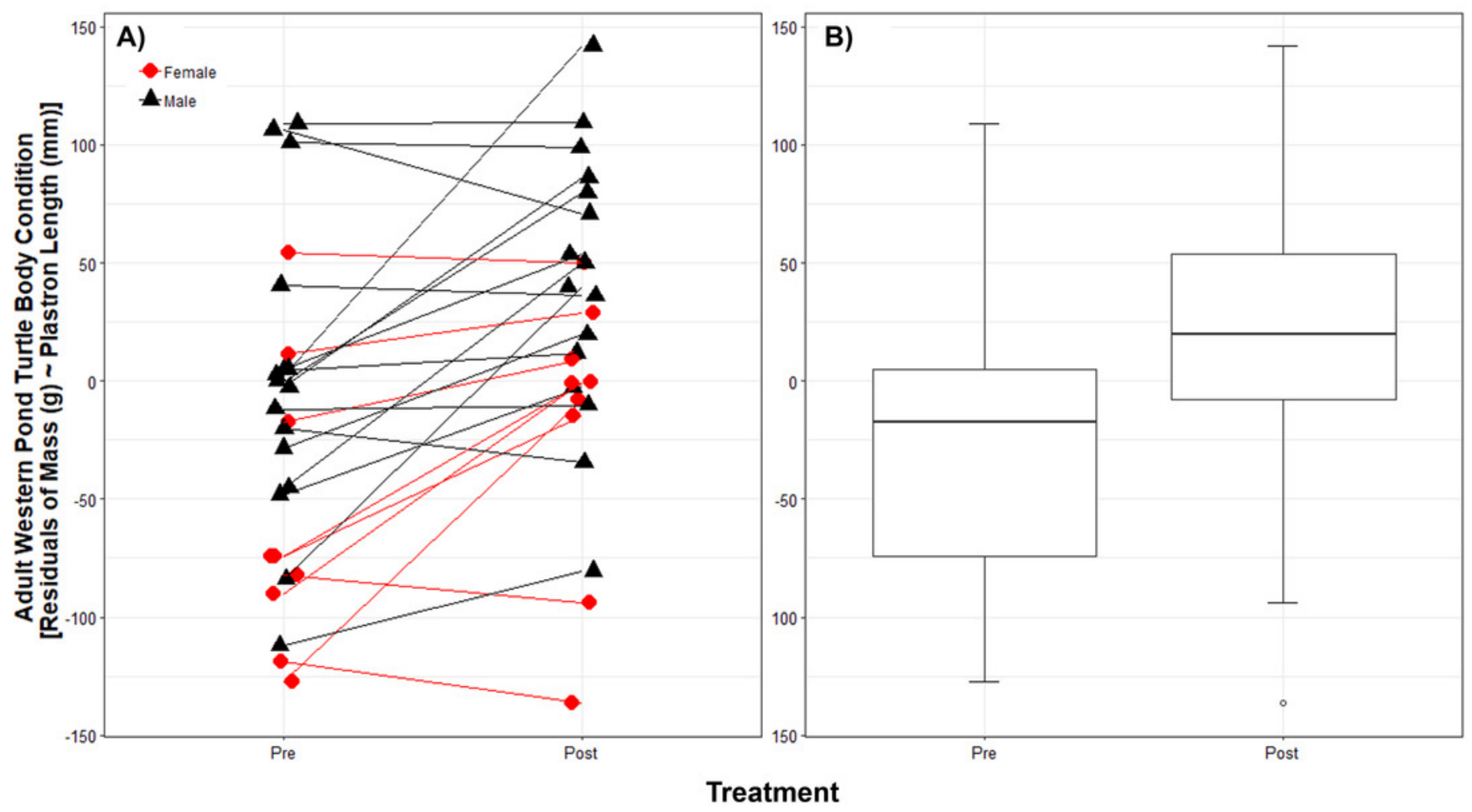


Figure 3

Cumulative basking observations of native WPT (Emys) and introduced RES

(Trachemys).

Basking observations before ( $\mathrm{A}$ ) and after (B) the RES removal are arrayed along a west-east gradient in the UCD Arboretum. Letters under the x-axis are basking site identifiers. Note the $y$-axes are on different scales in the two panels. 
A)

\section{Pre-Removal (2010)}

-Emys aTrachemys

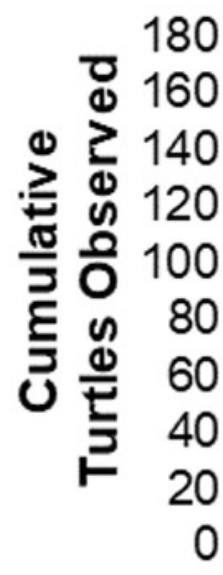

0

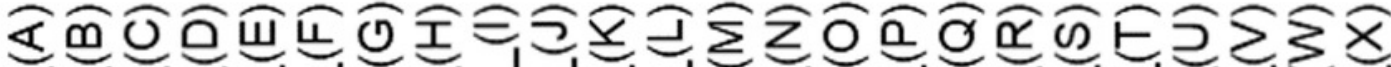

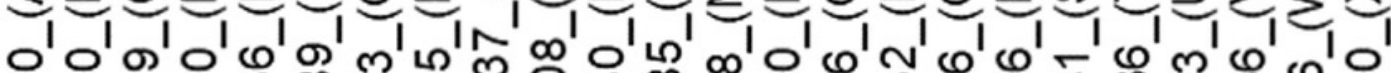
규 에 స N N

B)

\section{Post-Removal (2012)}

Emys aTrachemys

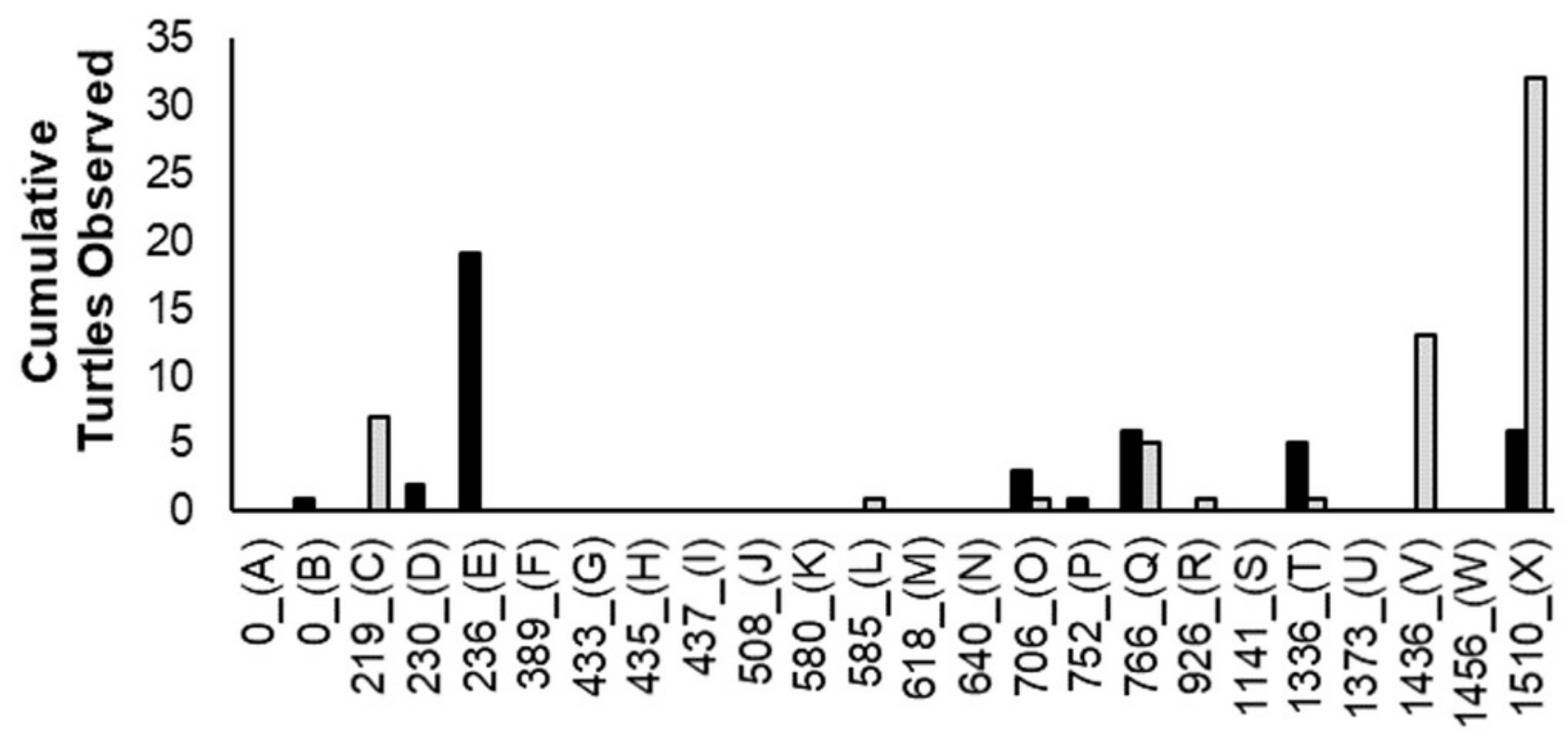

Distance (m) from West End 


\section{Figure 4}

Relative basking distribution of native WPT (Emys) to introduced RES (Trachemys) basking across the UCD Arboretum waterway pre- and post-removal.

Curves are the modeled ratios of WPT to RES basking along a west-east gradient in the UCD Arboretum pre- and post-removal (black and red curves, respectively). Models are for the relative basking distribution of the two species and account for the binary nature of these data and variation in sample sizes across basking sites and survey dates. The ratio of WPT to RES basking along the waterway was similarly WPT-biased in the west and RES-biased in the east in both years. WPT basking observations were higher after the RES removal. 


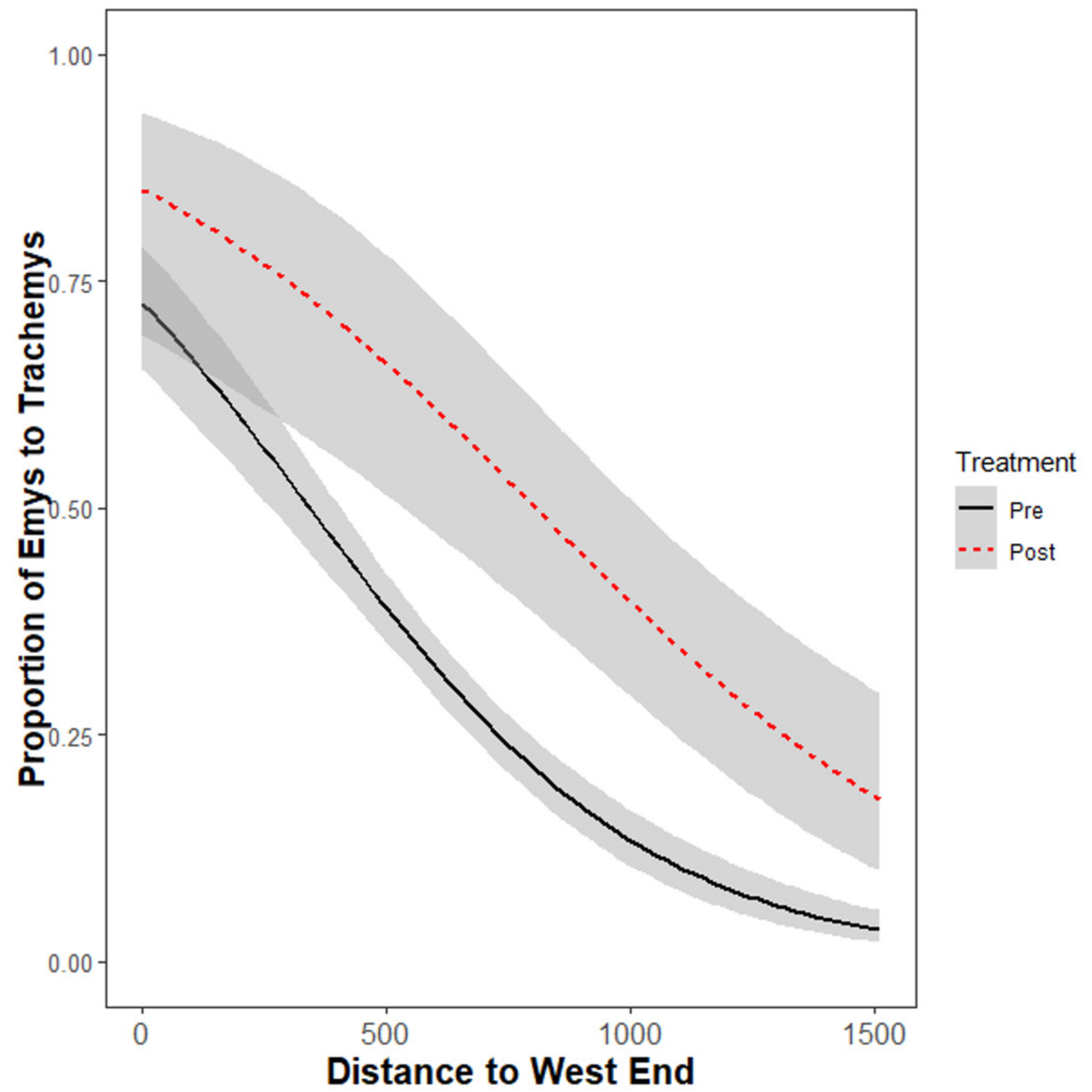


Figure 5

The total number of native WPT (Emys) and introduced RES (Trachemys) basking along the UCD Arboretum.

Curves are the modeled daily number of WPT and RES along a west-east gradient in the UCD Arboretum pre- and post-removal (black and red curves, respectively). WPT (A) displayed a more even basking distribution after the RES removal, and RES (B) concentrated basking activity towards the east end of the Arboretum after most of their population was removed.

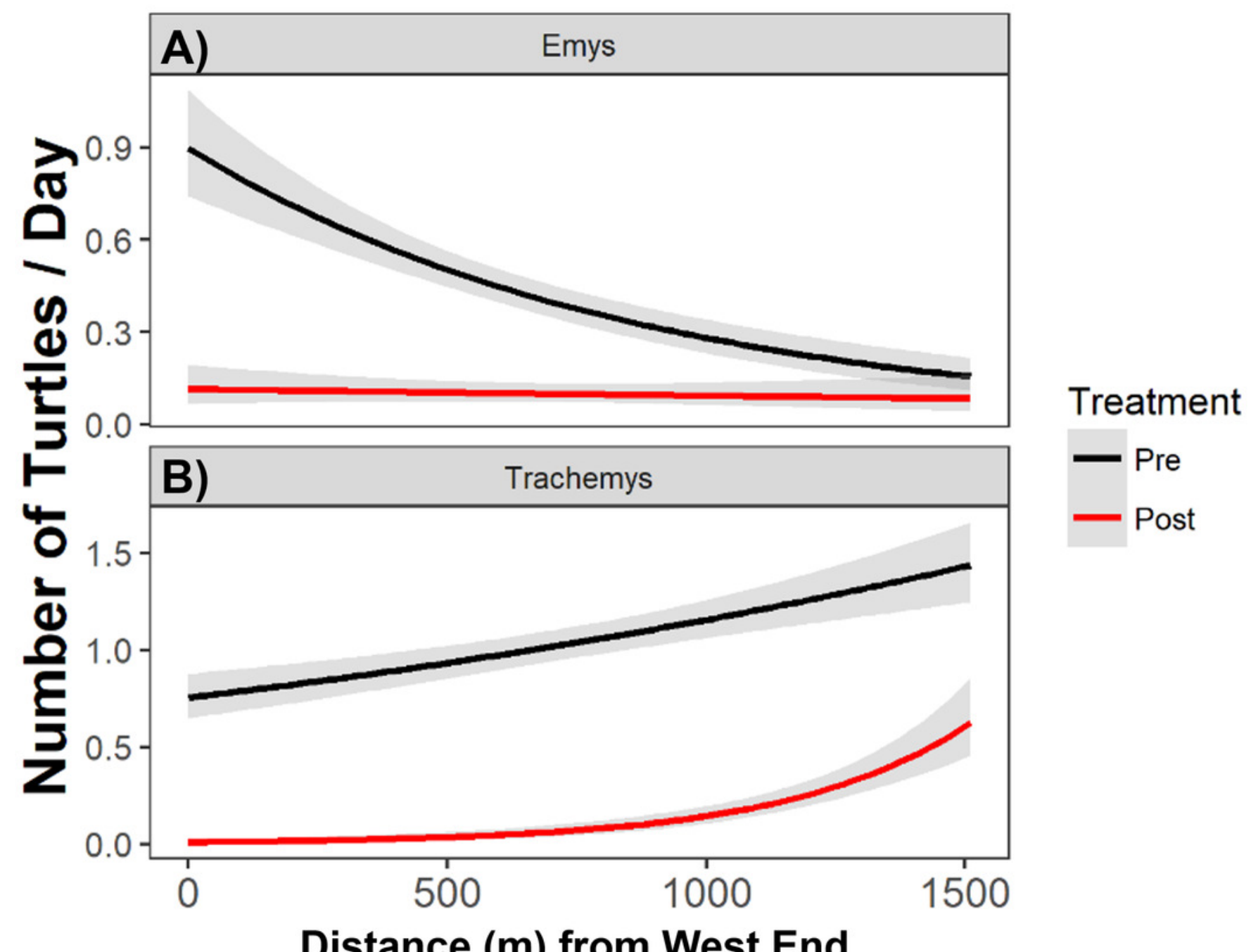

